\title{
Impact of $A_{0}$ on the mSUGRA parameter space \& Consequences for Cold Dark Matter Searches
}

\author{
Luisa Sabrina Stark ${ }^{1}$, Petra Häfliger ${ }^{1,2 *}$, Adrian Biland ${ }^{1}$ and Felicitas Pauss ${ }^{1}$ \\ ${ }^{1}$ Institute for Particle Physics, ETH Zürich, CH-8093 Zürich, Switzerland \\ ${ }^{2}$ Paul Scherrer Institute, CH-5232 Villigen PSI, Switzerland \\ E-mail: lstark@particle.phys.ethz.ch, petra.haefliger@phys.ethz.ch, \\ bilandeparticle.phys.ethz.ch, felicitas.paussecern.ch
}

\begin{abstract}
In minimal Supergravity (mSUGRA) models the lightest supersymmetric (SUSY) particle (assumed to be the lightest neutralino $\chi_{1}^{0}$ ) provides an excellent cold dark matter (CDM) candidate. The supersymmetric parameter space is significantly reduced, if the limits on the CDM relic density $\Omega_{C D M} h^{2}$, obtained from WMAP data, are used. Assuming a vanishing trilinear scalar coupling $A_{0}$ and fixed values of $\tan \beta$, these limits result in narrow lines of allowed regions in the $m_{0}-m_{1 / 2}$ plane, the so called WMAP strips. In this analysis the trilinear coupling $A_{0}$ has been varied within $\pm 4 \mathrm{TeV}$ resulting in largely extended areas in the $m_{0}-m_{1 / 2}$ plane which are no longer excluded.

This trilinear scalar coupling also influences the predicted $\gamma$-ray flux expected from neutralinopair annihilation and thus impacts on the possible detection of CDM using Cherenkov telescopes.
\end{abstract}

International Europhysics Conference on High Energy Physics

July 21st - 27th 2005

Lisboa, Portugal

*Speaker. 


\section{Introduction}

In the mSUGRA framework the lightest neutralino lends itself as an excellent CDM candidate, thus providing a connection between particle physics and astrophysics. The inclusion of cosmological experimental data allows to signifi cantly reduce the mSUGRA parameter space. The satellite born detector WMAP measured the abundance of CDM in the universe to be $0.094<\Omega_{C D M} h^{2}<$ 0.129 (at $2 \sigma$ C.L.) [1].

The SUSY parameter space in mSUGRA scenarios is usually studied in terms of the common scalar mass $m_{0}$, the common gaugino mass $m_{1 / 2}$, the ratio of the Higgs expectation values $\tan \beta$ and the sign of the Higgsino mass parameter $\mu$. However, the fi fth free parameter, the common trilinear scalar coupling $A_{0}$ was usually set to zero. In recent studies, the impact of non-zero $A_{0}$ values on the mSUGRA parameter space was recognised [2].

The soft SUSY breaking part of the Lagrangian provides additional contributions to the couplings of Higgs bosons to sfermions. However, as they are proportional to the mass of the corresponding SM fermion, they are only relevant for the third generation. These new couplings affect the masses of the sparticles through renormalisation group evolutions and through mixing effects. The relic density of the CDM particles, $\Omega_{C D M} h^{2}$, is connected to their annihilation cross sections by the LeeWeinberg equation. A variation of $A_{0}$ affects the effective cross section and therefore also the relic density through the dependence of the SUSY particle masses on these trilinear scalar couplings at the EW scale.

\section{The mSUGRA Parameter Space}
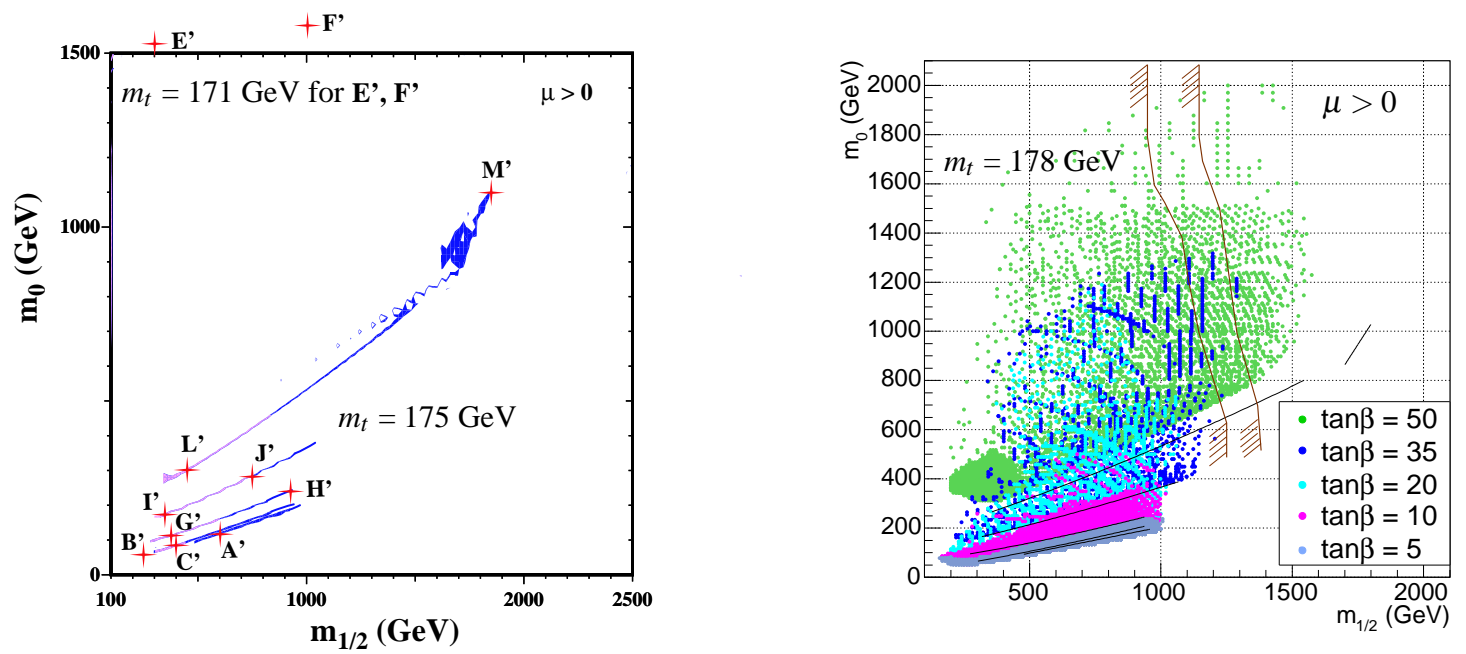

Figure 1: Allowed models for $m_{0}, m_{1 / 2} \leq 2 \mathrm{TeV}$, $\tan \beta$ between 5 and 50, $\mu>0$ after imposing the WMAP constraints on $\Omega_{\chi} h^{2}$. For the WMAP strips [3] in the left plot a vanishing trilinear scalar coupling was assumed, while $A_{0}$ was left free within $\pm 4 \mathrm{TeV}$ in the right one. The brown lines indicate the LHC discovery reach for an integrated luminosity of $100 \mathrm{fb}^{-1}$ and $300 \mathrm{fb}^{-1}$.

Assuming CDM to consist exclusively of the lightest neutralino, the cosmological bounds on the neutralino relic density $\Omega_{\chi} h^{2}$ imply constraints on the mSUGRA parameter space, which however also depend on $A_{0}$, as illustrated in Fig.1. Under the assumption of $A_{0}=0 \mathrm{TeV}$ and fi xed 
$\tan \beta$ values, only narrow lines in the $m_{0}-m_{1 / 2}$ plane (left plot in Fig.1) are left over as allowed regions after including WMAP data [3]. The right plot of Fig.1 illustrates the effect of varying $A_{0}$ within $\pm 4 \mathrm{TeV}$ [4]. In contrast to the left plot, where only a line survived the WMAP constraints for each chosen $\tan \beta$ value, extended regions in the mSUGRA parameter space are allowed for the same $\tan \beta$ value, if $A_{0}$ is varied.

\section{3. $\gamma$-Ray Flux from CDM annihilation}

In the following only the upper bound on the CDM relic density provided by WMAP is respected, as the total amount of CDM may not consist exclusively of neutralinos. The expected $\gamma$-ray flux is a function of the SUSY parameters and the halo properties of the observed target. The factor derived from particle physics is called ASUSY and includes the information about the number of photons produced per annihilation $N_{\gamma}$, the thermal average of the annihilation cross section times the velocity $\langle\sigma v\rangle$ and the neutralino mass $M_{\chi}$. The cosmological characteristics of the source enter in the calculation of the so called $\mathrm{J}(\psi)$ factor, which is the integral over the line of sight (los) of the CDM density distribution squared. $\psi$ is the angle between the direction of the target and that of observation.

(a)

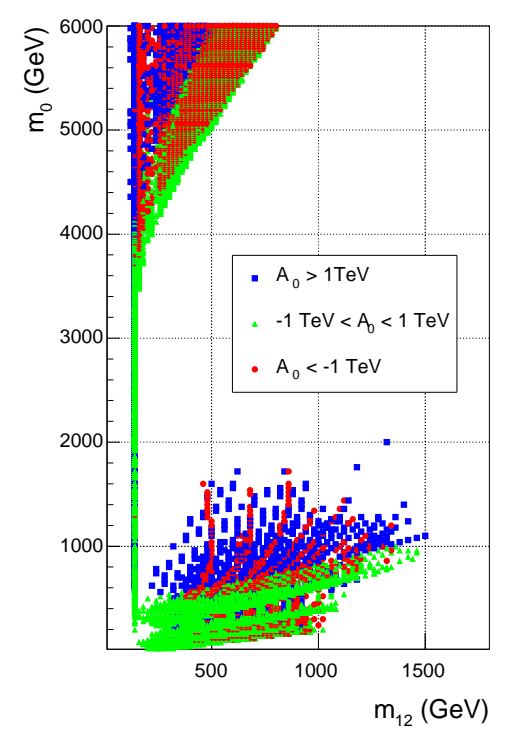

(b)

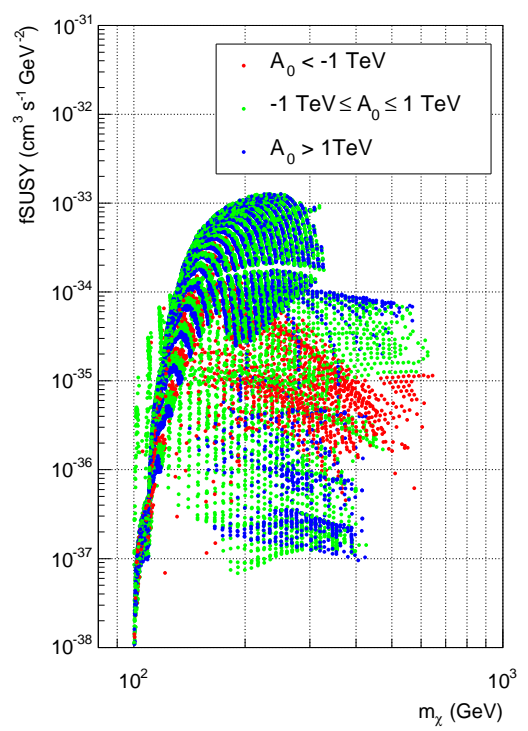

Figure 2: (a) Allowed mSUGRA models in the $m_{0}-m_{1 / 2}$ plane for different $A_{0}$ regions (all other parameters like in Fig.1) (b) The corresponding fSUSY factor for the generated models.

The properties of the SUSY model determine the fSUSY factor. Four different parameter regions in the $m_{0}-m_{1 / 2}$ plane exist (Fig.2a): The bulk region at low $m_{0}, m_{1 / 2}$ values, the coannihilation region with small $m_{0}$, the focus point region at low $m_{1 / 2}$ values and the region for very high $m_{0}$ values. mSUGRA models with $m_{0}$ values in the multi $\mathrm{TeV}$ region correspond to large fSUSY factors (Fig.2b). These models provide the highest $\gamma$-ray fluxes. However they only exist under the assumption that a small fraction of the total amount of CDM consists of neutralino.

Since the energy dependence of the neutralino CDM flux does not depend on the cosmological 
$\mathrm{J}(\psi)$ factor, the shape of the annihilation spectra is already defi ned by the fSUSY factor (Fig.3). The spectra of models with a small neutralino mass show a strong $A_{0}$ dependence for small values of $\tan \beta$.
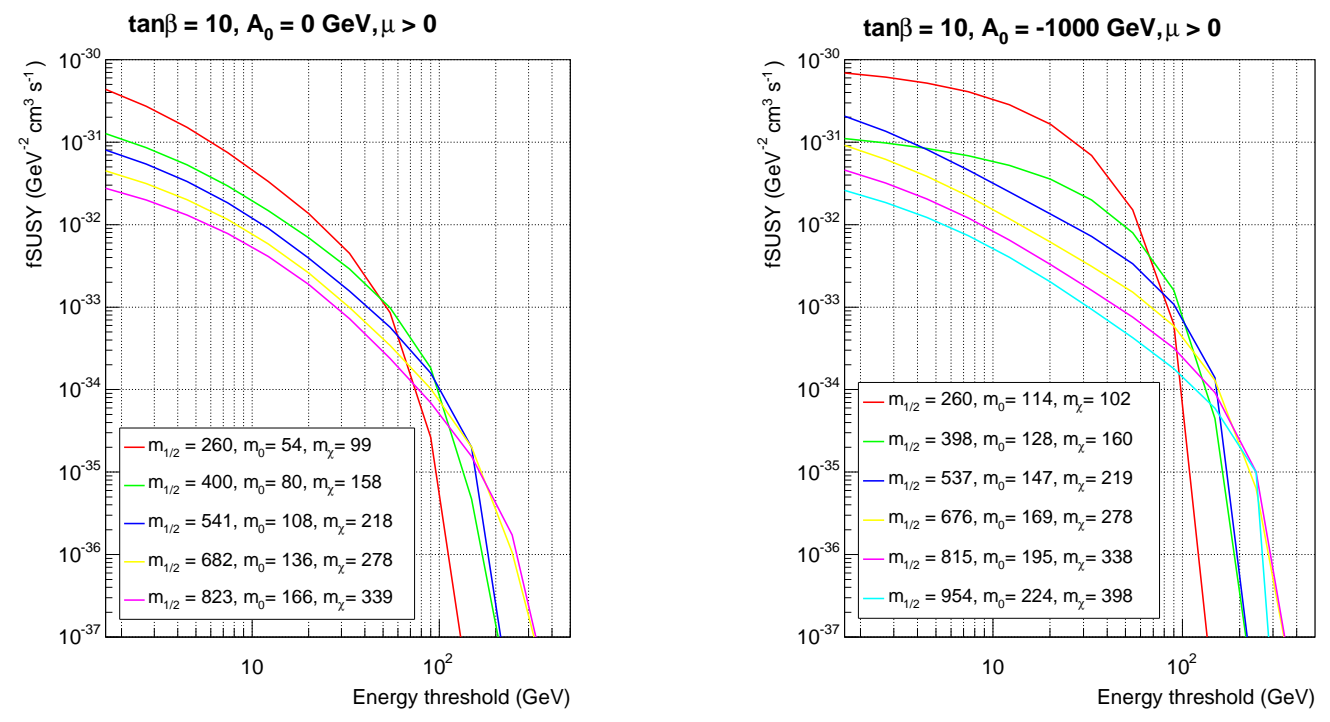

Figure 3: Annihilation spectra for mSUGRA models with $A_{0}=0 \mathrm{TeV},-1 \mathrm{TeV}$ and tan $\beta=10$.

\section{Conclusion}

The lightest neutralino in the MSSM is an excellent CDM candidate. Within the mSUGRA framework neutralino annihilation cross sections can be calculated. However, the predicted number of photons produced per annihilation as well as their energy spectrum depends strongly on the chosen parameters. Particular care must be taken to also include a possible non-zero trilinear scalar coupling $A_{0}$, resulting in an extended area of the allowed SUSY parameter space, after taking WMAP constraints into account. This also influences the discovery potential for CDM using space-born or ground-based instruments for photon detection.

\section{References}

[1] C. Bennett et al.. [WMAP Collaboration], Astrophys. J. Suppl. 148 (2003) 1, [astro-ph/0302207]; D.N. Spergel et al. [WMAP Collaboration], Astrophys. J. Suppl. 148 (2003) 175, [astro-ph/0302209].

[2] V. Bertin, E. Nezri and J. Orloff, Eur. Phys. J. C26 (2002) 111, [hep-ph/ 0204135$]$; J.R. Ellis, S. Heinemeyer, K.A. Olive and G. Weiglein, JHEP 0502 (2005) 013, [hep-ph/ 0411216 ].

[3] J.R. Ellis, K.A. Olive, Y. Santoso, V.C. Spanos, Phys. Lett. B565 (2003) 176, [hep-ph/ 0303043 ].

[4] L. S. Stark, P. Häfliger, A. Biland and F. Pauss, JHEP, 0508 (2005) 059, [hep-ph/ 0502197$].$

[5] A. Tasitiomi and A.V. Olinto, Phys. Rev. 66 (2002) 083006, [ast ro-ph / 0206040 ]. 